\title{
VISION IMPAIRMENT IN CHILDREN WITH CEREBRAL PALSY: CHARACTERISTICS AND TRENDS IN 1976-1998. A EUROPEAN REGISTER BASED STUDY
}

J. De La Cruz ${ }^{1}$, S. Wariyar ${ }^{2}$, D. Lora ${ }^{1}$, E. Sellier ${ }^{3}$, C.R. Pallás ${ }^{4}$, C. Cans ${ }^{3}$, A. Colver ${ }^{5,6}$, SCPE Collaboration

${ }^{I}$ Clinical Research Unit (IMAS12-CIBERESP), 12 de Octubre University Hospital, SERMAS, Madrid, Spain,

${ }^{2}$ Paediatrics, Great North Children's Hospital, Newcastle upon Tyne, UK, ${ }^{3}$ TIMC/ThEMAS-RHEOP, Grenoble University Hospital, Grenoble, France, ${ }^{4}$ Neonatology (IMAS12-SAMID), 12 de Octubre University Hospital, SERMAS, Madrid, Spain, ${ }^{5}$ Institute of Health and Society, Newcastle University, ${ }^{6}$ James Spence Building, Royal Victoria Infirmary, Newcastle, UK

Background and aims: Cerebral palsy (CP) is the most frequent cause of severe physical disability in children. Associate impairments are common in children with CP. The aims were to describe changes in prevalence of children with CP and vision impairment (VI), and to examine the association between VI and neonatal characteristics, associated impairments and CP subtypes.

Methods: All children with CP born 1976-1998 recorded in 17 European population based registers belonging to the SCPE network (Surveillance of Cerebral Palsy in Europe) were eligible. Data were coded according to SCPE Reference and Training Manual. Time trends were modelised with Poisson regression and adjusted for centre effect and study variables.

Results: Of the 9631 eligible children, 3300 (34\%) had a documented VI (AVI) and 966 (10\%) a known severe VI (SVI). A significant change in trend of prevalence of CP with AVI or SVI was observed with a moderate increase until end of the $80^{\prime}$ and a subsequent slight decrease. Children with $\mathrm{CP}$ born at term were less likely to have any VI (odds ratio $0.60(0.55-0.68))$ but no differences were observed for SVI $(0.97(0.81$ $1.15))$. Children with unilateral CP were less likely to show AVI (0.39 (0.35-044)) or SVI (0.17 (0.13-0.21)); those unable to walk even with aids were more likely to show AVI (4.8 (4.4-5.4)) or SVI (16 (14-21)).

Conclusions: Among children with $\mathrm{CP}$ one third shows any vision impairment and one in ten shows severe vision impairment. Vision impairment is associated with other impairments (intellectual, hearing) and epilepsy. 